\title{
APES Beamforming Applied to Medical Ultrasound Imaging
}

Blomberg, Ann E. A.; Holfort, Iben Kraglund; Austeng, Andreas; Synnevåg, Johan-Fredrik; Holm, Sverre; Jensen, Jørgen Arendt

Published in:

2009 IEEE International Ultrasonics Symposium (IUS)

Link to article, DOI:

10.1109/ULTSYM.2009.5441542

Publication date:

2009

Document Version

Publisher's PDF, also known as Version of record

Link back to DTU Orbit

Citation (APA):

Blomberg, A. E. A., Holfort, I. K., Austeng, A., Synnevåg, J-F., Holm, S., \& Jensen, J. A. (2009). APES

Beamforming Applied to Medical Ultrasound Imaging. In 2009 IEEE International Ultrasonics Symposium (IUS) (pp. 2347-2350 ). IEEE. https://doi.org/10.1109/ULTSYM.2009.5441542

\section{General rights}

Copyright and moral rights for the publications made accessible in the public portal are retained by the authors and/or other copyright owners and it is a condition of accessing publications that users recognise and abide by the legal requirements associated with these rights.

- Users may download and print one copy of any publication from the public portal for the purpose of private study or research.

- You may not further distribute the material or use it for any profit-making activity or commercial gain

- You may freely distribute the URL identifying the publication in the public portal 


\title{
APES Beamforming Applied to Medical Ultrasound Imaging
}

\author{
Ann E.A. Blomberg*, Iben Kraglund Holfort ${ }^{\dagger}$ Andreas Austeng*, Johan-Fredrik Synnevåg*, \\ Sverre Holm*, Jørgen Arendt Jensen ${ }^{\dagger}$, \\ *Department of Informatics, University of Oslo, P.O. Box 1080, NO-0316 Oslo, Norway \\ ${ }^{\dagger}$ Department of Electrical Engineering, Technical University of Denmark, DK-2800 Kgs. Lyngby, Denmark
}

\begin{abstract}
Recently, adaptive beamformers have been introduced to medical ultrasound imaging. The primary focus has been on the minimum variance (MV) (or Capon) beamformer. This work investigates an alternative but closely related beamformer, the Amplitude and Phase Estimation (APES) beamformer. APES offers added robustness at the expense of a slightly lower resolution. The purpose of this study was to evaluate the performance of the APES beamformer on medical imaging data, since correct amplitude estimation often is just as important as spatial resolution. In our simulations we have used a $3.5 \mathrm{MHz}$, 96 element linear transducer array. When imaging two closely spaced point targets, APES displays nearly the same resolution as the MV, and at the same time improved amplitude control. When imaging cysts in speckle, APES offers speckle statistics similar to that of the DAS, without the need for temporal averaging.
\end{abstract}

\section{INTRODUCTION}

The minimum variance (MV) beamformer was originally used in passive systems for e.g. direction finding [1], and later extended to active systems such as medical ultrasound imaging [2], [3], [4]. It has been shown that the MV beamformer, when used with the right parameters, significantly improves the image resolution compared to the conventional delay-and-sum (DAS) beamformer. However, the MV is sensitive to errors in the steering vector and also suffers from signal cancellation in the case of coherent sources. Diagonal loading [5] and subarray averaging [6] are necessary in order to address these issues. Still, the MV tends to under-estimate the amplitude of scatterers in some cases.

The closely related Amplitude and Phase Estimation (APES) beamformer was developed for improved amplitude control at the expense of slightly lower resolution. In [7], it is shown that APES is more robust against sound speed errors than MV when imaging single point targets. In this work, we have investigated the performance of the APES beamformer when imaging single and double point targets, as well as a cyst phantom. We have compared the APES beamformer to the MV and the DAS beamformers, with respect to resolvability, beamwidth, amplitude control and speckle appearance and statistics. In this context, resolvability is defined as the relative difference between the peak amplitude of the two laterally spaced point targets and the saddle point between them.

\section{Methods}

\section{A. The DAS beamformer}

In the conventional DAS beamformer, a time delay is applied to the received signal from each of the sensors to steer and focus the beam in a given direction, before coherently combining the signals. Given an array of $M$ sensors, the output of a general beamformer may be written as

$$
z[n]=\sum_{m=0}^{M-1} w_{m}^{*} y_{m}\left[n-\Delta_{m}\right],
$$

where $n$ denotes the time sample index and $\Delta_{m}$ is the time delay applied to sensor $m . y_{m}\left[n-\Delta_{m}\right]$ is the received and delayed signal at element $m$. The signal received at each sensor is multiplied by a weight, $w_{m}$. In conventional, nonadaptive beamformers such as DAS, these weights are predefined and thus data-independent. Often, the sensor weights are defined by a window function such as a Hanning or a Kaiser window.

\section{B. The MV beamformer}

The MV beamformer differs from the DAS beamformer in the way in which the weights, $w_{m}$ in (1), are calculated. Instead of using a set of pre-defined weights, the MV beamformer uses the recorded data field in order to compute the weights which minimize the variance of the output from the beamformer, while maintaining unit gain in the direction of interest. The MV beamformer computes the aperture weights by solving the following minimization problem [1]

$$
\min _{\mathbf{W}} \mathbf{w}[n]^{H} \hat{\mathbf{R}}[n] \mathbf{w}[n] \text { subject to } \mathbf{w}[n]^{H} \mathbf{a}=1,
$$

where $\mathbf{w}$ is an $M \times 1$ vector containing the complex sensor weights, $\hat{\mathbf{R}}$ is the estimated spatial covariance matrix, and $\mathbf{a}$ is the steering vector. Eq. (2) has an analytical solution given by

$$
\mathbf{w}_{M V}[n]=\frac{\hat{\mathbf{R}}[n]^{-1} \mathbf{a}}{\mathbf{a}^{H} \hat{\mathbf{R}}[n]^{-1} \mathbf{a}} .
$$

In active imaging systems, sub-array averaging is used to address the problem of signal cancellation caused by coherent sources. This involves dividing the transducer array into subarrays of length $L$, computing the spatial covariance matrix of each of the sub-arrays and using the averaged covariance matrix in (3). The parameter $L$ should be chosen with care, as discussed in [3] and [4]. While long sub-arrays result in improved resolution, shorter sub-arrays tend to give more robust amplitude estimates. Diagonal loading, i.e. adding a 
small amount of energy to the diagonal of the covariance matrix, is often necessary to ensure an invertible and robust estimate of the covariance matrix.

\section{The APES beamformer}

The APES beamformer is designed such that the output is as close as possible to a plane wave with wavenumber $k_{x}$, where $k_{x}$ represents the direction in which the beam is steered. The APES beamformer computes the weights which solve the following minimization problem

$$
\begin{aligned}
& \min _{\mathbf{W}, \alpha} \frac{1}{M-L+1} \sum_{m=0}^{M-L+1}\left|w^{H} y_{m}[n]-\alpha e^{j k_{x} x_{m}}\right|^{2} \\
& \text { subject to } \mathbf{w}[n]^{H} \mathbf{a}=1,
\end{aligned}
$$

where $n$ denotes the time sample index, $\mathbf{w}$ is a vector of apodization weights, $m$ denotes the element number, $x_{m}$ is the $\mathrm{x}$-coordinate of element $m$ and $\alpha$ is the complex amplitude of the desired plane wave. Let $G\left(k_{x}\right)=$ $\frac{1}{M} \sum_{m=0}^{M-1} y_{m}[n] e^{-j k_{x} x_{m}}$. The expression to be minimized in (4) can be re-written as [8]

$$
\begin{array}{r}
\frac{1}{M-L+1} \sum_{m=0}^{M-L}\left|w^{H} y_{m}[n]-\alpha e^{j k_{x} x_{m}}\right|^{2} \\
=\mathbf{w}^{H} \hat{\mathbf{R}} \mathbf{w}-\alpha^{*} \mathbf{w}^{H} \mathbf{G}\left(k_{x}\right)-\alpha \mathbf{G}^{H}\left(k_{x}\right) \mathbf{w}+|\alpha|^{2} \\
=\left|\alpha-\mathbf{w}^{H} \mathbf{G}\left(k_{x}\right)\right|^{2}+\mathbf{w}^{H} \hat{\mathbf{R}} \mathbf{w}-\left|\mathbf{w}^{H} \mathbf{G}\left(k_{x}\right)\right|^{2} .
\end{array}
$$

Minimizing (5) with respect to $\alpha$, gives $\hat{\alpha}=\mathbf{w}^{H} G\left(k_{x}\right)$. Inserting this in (5) results in the following minimization problem

$$
\min _{\mathbf{w}} \mathbf{w}^{H} \hat{\mathbf{Q}} \mathbf{w} \text { subject to } \mathbf{w}[n]^{H} \mathbf{a}=1,
$$

where $\hat{\mathbf{Q}}=\hat{\mathbf{R}}-\mathbf{G}\left(k_{x}\right) \mathbf{G}^{H}\left(k_{x}\right)$. Eq. (6) has a solution given by [8]

$$
\mathbf{w}_{A P E S}=\frac{\hat{\mathbf{Q}}^{-1} \mathbf{a}\left(k_{x}\right)}{\mathbf{a}^{H}\left(k_{x}\right) \hat{\mathbf{Q}}^{-1} \mathbf{a}\left(k_{x}\right)} .
$$

The APES solution is equivalent in form to the MV solution, with the exception that the spatial covariance matrix, $\hat{\mathbf{R}}$, is replaced by $\hat{\mathbf{Q}}$, which may be interpreted as the noise covariance matrix.

\section{RESUlTS AND DISCUSSION}

We have used Field II [9], [10] for the simulations. Data was obtained for point targets and for a cyst phantom in speckle, using a 96-element, $3.5 \mathrm{MHz}, 18.5 \mathrm{~mm}$ transducer. Dynamic focusing was used both on transmission and on reception. The cyst was modelled as a cylindrical area with a radius of $3 \mathrm{~mm}$, within which the reflection coefficient of the scatterers is zero. Homogeneous tissue surrounding the cyst region was modelled by placing 350000 randomly distributed point scatterers within a volume defined by a radial distance of 3 to $5 \mathrm{~cm}$, a lateral angle of $-90^{\circ}$ to $90^{\circ}$ and an elevation angle of $-3.9^{\circ}$ to $3.9^{\circ}$, corresponding to the sezond zero point of the array beampattern. Two strong point reflectors were also placed inside the tissue region. Diagonal loading corresponding to $\frac{5 \%}{L}$ of the received power was used, in accordance with previously recommended parameter sets [4].

Fig. 1 shows the steered response from two closely spaced point reflectors imaged using the DAS with uniform weighting (dashed line), the MV (solid line) and the APES (solid line with crosses) beamformer. The point reflectors are placed 3 $\mathrm{mm}$ apart, at a depth of $5 \mathrm{~cm}$. In the leftmost plot, a subarray length of $L=24$ is used, in the middle $L=36$ and on the right $L=48$. For all choices of $L$, The MV and APES bamformers display significantly narrower mainlobes than the DAS, as well as better resolvability. Between the MV and the APES beamformers, the MV displays the narrowest mainlobe and the highest resolvability. The performance of the MV and the APES beamformers are similar for short sub-arrays $(L=24)$. As the sub-array length increases, the performance of the APES beamformer stays relatively constant while the MV displays increasing resolvability and a narrower mainlobe, at the expense of less robust amplitude estimates (underestimated by up to $5 \mathrm{~dB}$ ).

In Fig. 2, the resolvability (left), full width at half maximum (FWHM) (middle), and normalized peak amplitude (right) for each of the beamformers are plotted for sub-array lengths ranging from 24 to 48 in steps of 4 . The DAS beamformer is able to resolve the two points by $17 \mathrm{~dB}$, while the APES beamformer resolves them by about $19 \mathrm{~dB}$. For the MV, the resolvability improves significantly with the sub-array length, resolving the points by about $20 \mathrm{~dB}(L=24)$ to $25 \mathrm{~dB}(L=48)$. The FWHM values for the APES and MV beamformers are both much smaller than for the DAS beamformer, but again the MV beamformer shows a slightly narrower mainlobe than the APES beamformer. The peak amplitude of the APES beamformer stays nearly constant and very close to that of DAS, while it drops down to $5 \mathrm{~dB}(L=48)$ below that of the DAS, for the MV.

In medical ultrasound imaging, resolution and contrast as well as reliable amplitude estimates are of importance. Also, the speckle pattern caused by scattering from micro-structures within the tissue may be of clinical interest. Fig. 3 shows a cyst phantom in speckle together with two strong point reflectors. The DAS beamformer with a rectangular window (a), and with a Hanning window (b) result in some smearing of the point reflectors, as expected due to the wide mainlobe of the conventional beamformer. The Hanning window in (b) reduces the sidelobes, resulting in less energy leakage into the cyst region, but the resolution is decreased as can be seen from the point reflectors. The MV beamformer (c) gives welldefined point targets, but there is considerable leakage into the cyst region, and the speckle pattern looks quite different from what could be expected. Temporal averaging (as in (d) where averaging over one pulse length is used) has been suggested as a way to improve the speckle statistics when using the MV [11]. The APES beamformer (e) results in a clear definition of the edges of the cyst as well as the point reflectors. Also, there is less energy leakage from sidelobes. The increased amplitude 

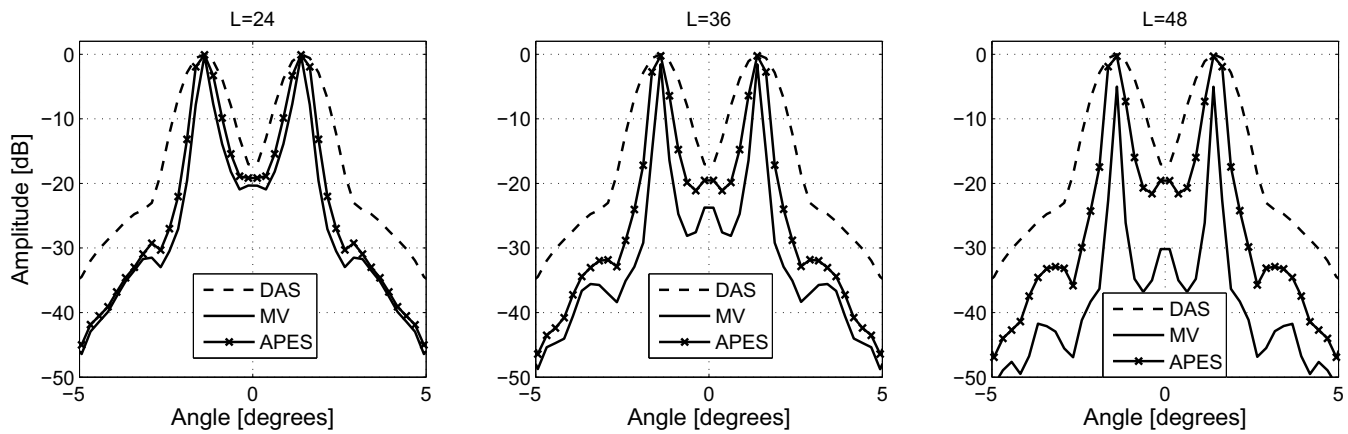

Figure 1: Steered response for the DAS (dashed), the MV (solid) and the APES (solid with crosses) beamformers, with L=24 (left), L=36 (middle) and $L=48$ (right).A $3.5 \mathrm{MHz} 96$ element array was used. The point targets are placed at a depth of $5 \mathrm{~cm}$.
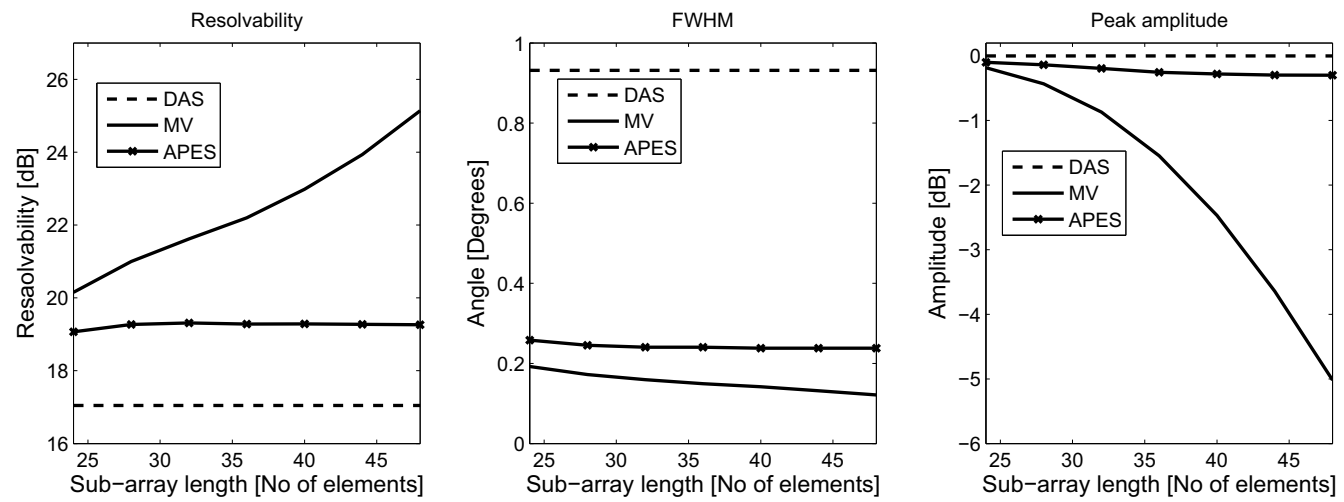

Figure 2: Resolvability (left), FWHM (middle) and normalized peak amplitude (right) for sub-array lengths from 24 to 48 in steps of four. The resolvability is measured as the relative distance between the peaks of two scatterers and the saddle point between them. FWHM is measured from the response of a single point scatterer. The peak amplitude is computed for double point reflectors ans is normalized by the peak amplitude of the DAS beamformer.
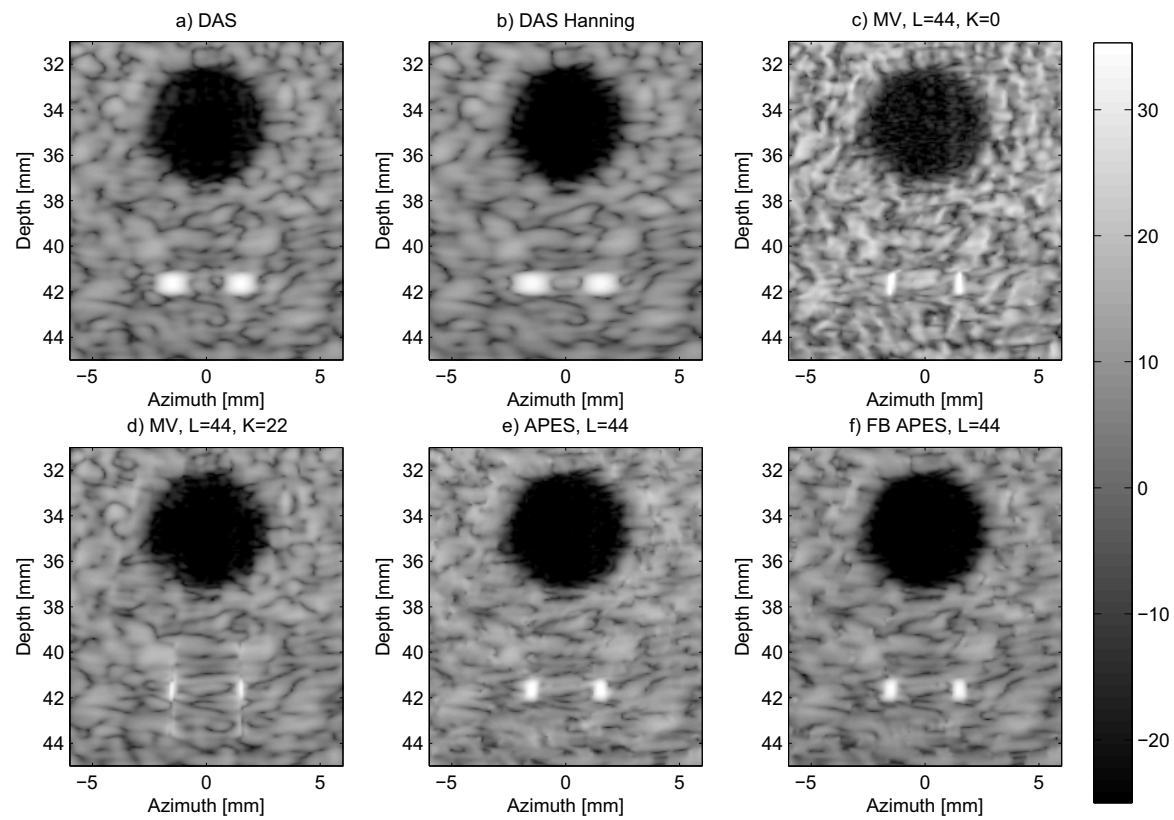

Figure 3: A simulated cyst phantom in speckle together with two strong reflectors, imaged using a 96 element, $18.5 \mathrm{~mm}, 3.5 \mathrm{MHz}$ transducer. Each image is normalized by its mean speckle value. (a): DAS with a rectangular window, (b): MV with temporal averaging over one pulse length, (c): MV with temporal averaging over one pulse-length, (d): APES, (e): Forward-backward APES. 
control of the APES beamformer results in a speckle pattern similar to that of the DAS beamformer, without the need for temporal averaging. Forward-backward averaging has been proposed by several authors, see e.g. [12], in order to further improve the APES estimate. APES with forward-backward averaging is shown in e).

To ensure stable results, we have run 100 simulations on a cluster of Linux workstations, randomly distributing the point scatterers each time. Fig. 4 shows a horizontal slice through the center of the cyst, created by averaging the images from the 100 simulations. 15 depth samples were averaged for each of the beamformers ((a), (d), and (e) from Fig. 3). Among these beamformers, APES provided the lowest value inside the cyst region together with the MV with temporal averaging, indicating a low amount of energy leakage into this area.

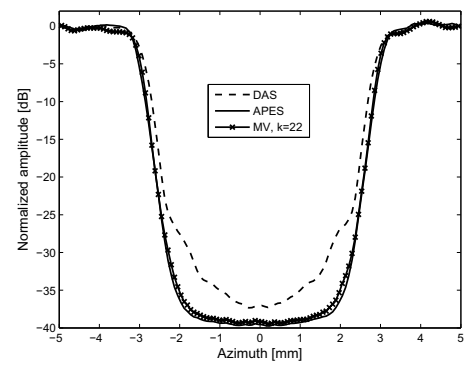

Figure 4: Horizontal slice through the center of the cyst, imaged using DAS rectangular window (dashed), APES (solid) and MV with temporal averaging (solid with crosses). Images from 100 simulations were averaged.

Speckle statistics are quantified using the pixel signal-tonoise ratio $\left(\mathrm{SNR}_{p}\right)$ (1.91 for fully developed speckle), defined as the mean value divided by the standard deviation within a speckle region [13]. Table I summarizes these values for each of the beamformers. The statistics in Table I are average values from the 100 simulations. These measurements show that the APES beamformer offers speckle statistics close to the theoretical value for fully developed speckle, without the need for temporal averaging. For the MV beamformer, temporal averaging over one pulse length is necessary in order to achieve speckle statistics comparable to that of the DAS.

\begin{tabular}{|l|c|c|r|}
\hline Beamformer & mean & std & $S N R_{p}$ \\
\hline \hline DAS & 0.31 & 0.16 & 1.91 \\
\hline DAS Hanning & 0.31 & 0.12 & 1.91 \\
\hline MV, K=0 & 0.16 & 0.13 & 1.21 \\
\hline MV, K=22 & 0.30 & 0.16 & 1.90 \\
\hline APES & 0.28 & 0.14 & 1.91 \\
\hline FB APES & 0.29 & 0.15 & 1.91 \\
\hline
\end{tabular}

Table I: Speckle statistics (mean, standard deviation and $S N R_{p}$ ) computed from a speckle region for each of the beamformers. The values are averaged over 100 realizations of speckle.

\section{CONCLUSION}

The APES beamformer may be preferable to the MV beamformer in applications where robustness and reliable amplitude estimates are of importance. The MV beamformer can achieve higher resolution than the APES beamformer, but it suffers from sensitivity to assumed parameters such as the propagation velocity, the choice of sub-array length and the amount of diagonal loading applied. The MV beamformer also suffers from signal cancellation in the presense of coherent sources, which is the case in an active system such as medical ultrasound imaging. Because of the way the minimization criterion is formulated in the APES beamformer, it does not suffer from signal cancellation to the same extent [12]. Also, the APES beamformer is less dependent on the choice of model parameters, and the improved amplitude control results in speckle patterns similar to that of the DAS beamformer. The computational burden of the APES beamformer is only marginally larger than that of the MV beamformer. The most demanding computational step of the MV and APES beamformers, is the matrix inversion in (3) and (7). Since the dimensions of the matrices $\hat{\mathbf{R}}$ and $\hat{\mathbf{Q}}$ are directly proportional to the sub-array lengths, $L$, using a shorter sub-array length will reduce the computational burden. As illustrated by Fig. 2, the performance of the APES beamformer is less dependent on $L$, so the sub-array length may be reduced without sacrificing performance. The fact that temporal averaging is unnecessary further reduces the number of computations. The APES beamformer is therefore a strong candidate for applications in medical ultrasound, offering high image resolution and contrast as well as robustness and speckle patterns similar to that of the DAS beamformer.

\section{REFERENCES}

[1] J. Capon. High-resolution frequancy-wavenumber spectrum analysis. Proc. IEEE, pages 1408-1418, August 1969.

[2] I. K. Holfort, F. G., and J. A. Jensen. Broadband minimum variance beamforming for ultrasound imaging. IEEE Trans. on Ultrason., Ferroelec., and Freq. Contr., February 2009.

[3] J.-F. Synnevåg, A. Austeng, and S. Holm. Adaptive beamforming applied to medical ultrasound imaging. IEEE Trans. Ultrason., Ferroelec., Freq. Contr., pages 1606-1613, August 2007.

[4] J.-F. Synnevåg, A. Austeng, and S. Holm. Benefits of minimum variance beamformning in medical ultrasound imaging. IEEE Trans. Ultrason., Ferroelec., Freq. Contr., September 2009.

[5] T.-J. Shan, M. Wax, and T. Kailath. On spatial smoothing for directionof-arrival estimation of coherent signals. IEEE Trans. Acoust., Speech, Signal Process., pages 806-811, August 1985.

[6] T.-J. Shan and T. Kailah. Adaptive beamforming for coherent signals and interference. IEEE Trans. Acoust., Speech, Signal Process., pages 527-536, June 1985.

[7] I. K Holfort, F. Gran, and J. A. Jensen. Investigation of sound speed errors in adaptive beamforming. In Proc. IEEE Ultrason. Symp., 2008.

[8] P. Stoica, H. Li, and J. Li. A new derivation of the apes filter. IEEE Signal Processing Letters, August 1999.

[9] J. A. Jensen and N. B. Svendsen. Calculation of pressure fields from arbitrarily shaped, apodized, and excited ultrasound transducers. IEEE Trans. Ultrason., Ferroelec., Freq. Contr., pages 262-267, 1992.

[10] J. A. Jensen. A program for simulating ultrasound systems. Med. Biol. Eng. Comp., vol. 10th Nordic-Baltic Conference on Biomedical Imaging, pages 351-353, 1996

[11] J.-F. Synnevåg, C. I. C. Nilsen, and S. Holm. Speckle statistics in adaptive beamforming. Proc. IEEE Ultrason. Symp., pages 1545-1548, October 2007.

[12] A. Jakobsson and P. Stoica. On the forward-backward spatial APES. Signal Process., 86(4):710-715, 2006.

[13] J. M. Thijssen, G. Weijers, and C. L. de Korte. Objective performance testing and quality assurance of medical ultrasound equipment. Ultrasound in Med. \& Biol., pages 460-471, March 2007. 